\title{
Joint Transmitter-Receiver Optimization in the Downlink CDMA Systems
}

\author{
Mohammad Saquib \\ Wireless Communications Research Lab. (WiCoRe), Department of Electrical Engineering, University of Texas at Dallas, \\ Richardson, TX 75083-0688, USA \\ Email: saquib@utdallas.edu \\ Md Habibul Islam \\ Wireless Communications Research Lab. (WiCoRe), Department of Electrical Engineering, University of Texas at Dallas, \\ Richardson, TX 75083-0688, USA \\ Email: hiron@utdallas.edu
}

Received 23 August 2001 and in revised form 15 March 2002

\begin{abstract}
To maximize the downlink code-division multiple access (CDMA) system capacity, we propose to minimize the total transmitted power of the system subject to users' signal-to-interference ratio (SIR) requirements via designing optimum transmitter sequences and utilizing linear optimum receivers (minimum mean square error (MMSE) receiver). In our work on joint transmitter-receiver design for the downlink CDMA systems with multiple antennas and multipath channels, we develop several optimization algorithms by considering various system constraints and prove their convergence. We empirically observed that under the optimization algorithm with no constraint on the system, the optimum receiver structure matches the received transmitter sequences. A simulation study is performed to see how the different practical system constraints penalize the system with respect to the optimum algorithm with no constraint on the system.
\end{abstract}

Keywords and phrases: CDMA system, joint transmitter-receiver optimization, MMSE receiver, power control, downlink, multipath and multiple antennas.

\section{INTRODUCTION}

Code-division multiple access (CDMA) systems are being considered to support multimedia traffic in the next generation mobile radio systems, such as CDMA2000 and WCDMA. Voice-based CDMA systems are generally equal in their uplink and downlink traffic, whereas, in future CDMA systems, which will support various types of high data rate image and video traffic with voice messages, the downlink will carry the significant portion of the total system traffic. Therefore, an important area of research is to maximize the downlink capacity via fully utilizing the limited system resources.

The capacity of a CDMA system is interference limited. Techniques that control or avoid interference improve the CDMA system capacity. There are three means of controlling interference in a CDMA system: power control, multiuser detection, and beamforming. Power control balances received powers of all users so that no user suffers from excessive interference due to other users in the system. Multiuser detection suppresses interference by exploiting the temporal structure of the interference, whereas beamforming uses the spatial structure of the interference to cancel it.

Recently, several studies $[1,2,3,4]$ have been performed in order to integrate power control with multiuser detection. The motivation of these works was to achieve a performance gain over multiuser detection by providing power control for multiuser detection. In $[1,3]$, the problem of finding the jointly optimum powers and linear receivers in synchronous CDMA systems are addressed. It is shown that a distributed and iterative power control algorithm, where each user employs linear minimum mean square error (MMSE) receiver filter before each power control update, converges to the point where all users spend minimum transmit powers and use corresponding MMSE receivers. The empirical results of [3] indicate that the linear MMSE receiver with optimum power allocation to users can significantly improve the system capacity.

In [5] three basic interference management approaches are combined, transmit power control, multiuser detection, and beamforming to increase the uplink capacity of a synchronous CDMA system. Due to the fact that in a CDMA 
system, a transmitter is a combination of power, spreading sequences, and beamforming weights, joint power-receiver optimization is suboptimum with respect to the joint transmitter and receiver optimization. Since it is most desirable to fully maximize the system capacity, in this work, we develop several joint transmitter-receiver optimization algorithms for CDMA systems with multipaths and multiple antennas under different practical system constraints.

Joint transmitter-receiver optimization is proposed in [6] for multiuser systems over multipath channels. Jang et al. [6] consider a single antenna at the base station and the meansquared error between the true bit value, and its estimate is taken as the cost function subject to average and peak power constraints. The capacity of a wireless system is interferencelimited and the interference in the system will be minimized if the transmitter powers are minimized. Therefore, we would like to develop joint transmitter-receiver optimization algorithm that minimizes the sum of the total transmitted power by all the transmitters subject to the SIR requirement of each user. Thus, the problem we are interested in solving is different from the problem addressed in [6].

The novel idea of multiple-access interference (MAI) elimination for CDMA systems by means of signature sequence adaptation has been considered in $[7,8,9,10]$. In [7], we see that it is possible to obtain the maximum information theoretic capacity [11] as well as user capacity [9] by developing optimal set of transmitter spreading sequences for synchronous CDMA systems. The user capacity is defined as the maximum number of supportable users at a common SIR target of a fixed processing gain CDMA system. For the synchronous CDMA system, signature sequence sets having the least total squared correlation (TSC) are the optimum in all cases [7]. In [8] an algorithm similar to [7] is developed, which yields the optimum sequences for asynchronous CDMA systems that achieve a lower bound on the total squared asynchronous correlation (TSAC) among the users. Here it is found that under the optimum signature sequences the user capacity of a single-cell asynchronous CDMA system is the same as that of a single-cell synchronous CDMA system. Recently, in [12] an algorithm analogous to [7, 8] is proposed to design joint transmitter power and spreading sequences for the uplink CDMA systems with multipath channels. Here it is assumed that all the users have the common SIR requirement and the proposed algorithm in [12] is suboptimum in multipath channels.

It should be mentioned that the transmitter beamforming with multielement transmit antenna arrays [13, 14] is also related to the signature sequence design. However, the key difference between transmit beamforming in multielement antenna arrays and signature sequence design in CDMA systems is that the spreading (temporal) sequences of the users in CDMA systems can be fully controlled by the transmitter, whereas, since the beamforming weights in multielement antenna systems are created by the wireless communications channel, they cannot be directly controlled by the transmitters. Recently, for CDMA systems with multimedia services, a downlink beamforming technique that converts the downlink beamforming problem into a virtual uplink one by taking into account the data rate information of all users is proposed [15].

In this work, we formulate the transmitter and receiver design problem as a joint optimization problem. We develop the optimum transmitter sequences by minimizing the cost function of the optimization problem, which is the total transmitted power by the system. The maximization problem in the constraint of the minimization problem yields the linear optimum receivers of users subject to their SIR constraints. We consider multipath CDMA systems with multiple antennas at the transmitter and a single antenna element at each receiver. As a consequence, our designed transmitter sequences are a combination of spreading sequences and optimum beamforming weights (spatial sequences). Practical constraints in the systems lead us to develop two constrained joint transmitter-receiver optimization algorithms for the downlink CDMA systems. Finally, it should be mentioned that the joint transmitter-receiver optimization problem in the uplink is not much different from that in the downlink.

In [14], Visotsky and Madhow has studied joint transmitreceive beamforming with the objective of minimizing the total transmitter power by the system subject to SIR requirements of users in space division multiple access (SDMA) systems. In SDMA systems, transmitters are modeled by antenna (beamforming) weights only. The differences between this work and [14] are that we concentrate on multipath CDMA systems, where a transmitter is a combination of power, temporal, and spatial sequences. We formulate a joint transmitter-receiver optimization problem with no constraint on the system and provide an iterative algorithm to solve it. Considering different practical system constraints, we also develop two other constrained joint transmitterreceiver optimization algorithms and prove their convergence.

The rest of this paper is organized as follows. Section 2 derives a vector model for a multipath single-cell CDMA system, where a transmitter broadcasts signals to many users by using multiple antennas. In Section 3, we propose the optimization algorithms that yield the transmitter and receiver structures of users under various system constraints. Section 4 shows the convergence of the proposed algorithms. Some empirical results and final remarks are presented in Sections 5 and 6 , respectively.

\section{SYSTEM MODEL}

Consider a CDMA system that has one transmitter and many receivers, where each receiver is equipped with a single antenna element and the transmitter broadcasts signals to receivers using an $M$ element antenna array. This CDMA system model could be applied to the downlink of WCDMA [16] or that of Infostations, which is an array of isolated wireless ports proposed in [17] to provide convenient and frequent access to a wide range of useful and economical Internet type services. There are $K$ receivers in the system and, without loss of generality, receiver $q$ is the receiver of interest. We assume that each receiver corresponds to a unique 
user and thus receiver $k$ and user $k$ are one in the same. It is assumed that a single transmitter employs $M$ antennas transmitting asynchronously over $M$ different multipath channels to each receiver. Here, the transmitter has accurate feedback from receivers regarding transmitter-receiver channels seen by the receivers and it executes the proposed algorithms. It is also the responsibility of the transmitter to inform the receivers of their coefficients.

At each transmit antenna, each bit results in the transmission of a sequence of pulses, or chips, $p[t]$, where each pulse has a duration of one chip period $T_{c}$. The bit transmission time is $T$ and the processing gain is $L=T / T_{c}$. Slow fading is assumed on each channel. We also assume that the maximum delay spread is very small with respect to $T$ and thus we ignore intersymbol-interference (ISI). The length of the observation window is $D T_{c}$, where $D \geq L$ is an integer. In this work, $\mathbf{X}^{\mathrm{T}}$ denotes the transpose of the matrix $\mathbf{X}$ and $\mathbf{X}^{\mathrm{H}}$ the Hermitian of the matrix $\mathbf{X}$.

Let $a_{k}^{(m, i)}$ be the $m$ th chip for user $k$ at antenna $i$; we use $\left\{a_{k}^{(m, i)}\right\}$ to denote the set of temporal or spreading sequences for user $k$. The temporal sequence vector for user $k$ at antenna $i$ is $\mathbf{a}_{k}^{(i)}=\left[a_{k}^{(1, i)}, \ldots, a_{k}^{(L, i)}\right]$. Using $\mathbf{O}(n)$ to denote a zero row vector of size $n$, we can simplify the description of the received signal by defining the $M L \times 1$ vector

$$
\mathbf{A}_{k}^{(i)}=\left[\mathbf{O}((i-1) L), \mathbf{a}_{k}^{(i)}, \mathbf{O}((M-i) L)\right]^{\mathrm{T}} .
$$

Using $\mathbf{A}_{k}^{(i)}$, we define

$$
\mathbf{A}_{k}=\left[\mathbf{A}_{k}^{(1)}, \ldots, \mathbf{A}_{k}^{(M)}\right]
$$

where $\mathbf{A}_{k}$ is an $M L \times M$ matrix. For each receiver $k$, the transmitter employs an antenna weight vector

$$
\mathbf{w}_{k}=\left[w_{k}^{(1)}, \ldots, w_{k}^{(M)}\right]^{\mathrm{T}},
$$

where $w_{k}^{(i)}$ is the weight used at antenna $i$ for user $k$. We call $\left\{w_{k}^{(i)}\right\}$ the set of spatial sequences for user $k$. Using (2) with (3), we define $\boldsymbol{S}_{k}$ as

$$
\mathbf{S}_{k}=\mathbf{A}_{k} \mathbf{w}_{k} .
$$

The term $\mathbf{S}_{k}$ denotes a column vector of size $M L$ and its elements from $(i-1) L+1$ to $i L$ are the joint temporal-spatial sequences that have been employed at antenna $i$ for receiver $k$. The total transmitter power for receiver $k$ is $\left\|\mathbf{S}_{k}\right\|^{2}=P_{k}$. In [18], we show that at the end of a bit interval, the chip matched filter outputs of user $q$ yield the received signal vector

$$
\mathbf{R}_{q}=\sum_{k=1}^{K} b_{k} \mathbf{H}_{q} \mathbf{A}_{k} \mathbf{w}_{k}+\mathbf{N}=\sum_{k=1}^{K} b_{k} \mathbf{H}_{q} \mathbf{S}_{k}+\mathbf{N}
$$

where $b_{k} \in\{-1,1\}$ is the transmitted bit for receiver $k, \mathbf{H}_{q}$ is a $D \times M L$ matrix whose components are functions of parameters of $M$ multipath channels and the structure of the transmitter pulse $p[t]$. If the contribution of the $i$ th antenna to $\mathbf{H}_{q}$ is $\mathbf{H}_{q}^{(i)}$, then

$$
\mathbf{H}_{q}=\left[\mathbf{H}_{q}^{(1)}, \ldots, \mathbf{H}_{q}^{(M)}\right],
$$

where the size of $\mathbf{H}_{q}^{(i)}$ is $D \times L$. The term $\mathbf{N}$ is a complex white Gaussian noise vector with zero mean and covariance

$$
E\left[\mathbf{N N}^{\mathrm{H}}\right]=\sigma^{2} \mathbf{I}_{D}
$$

where $\mathbf{I}_{D}$ is an identity matrix of size $D \times D$.

\section{JOINT TRANSMITTER-RECEIVER OPTIMIZATION}

To maximize the system capacity, we will minimize the total power transmitted by the system subject to quality of service $(\mathrm{QoS})$ requirements of users, where QoS is defined in terms of the SIR. Here, our main assumption for developing jointly optimum transmitters and receivers for CDMA systems is that the transmitters and receivers have the accurate information that they need to operate.

The detection of the information bit of the desired receiver $q$ is done by taking the sign of the decision statistics, which is to be found, using the observation vector $\mathbf{R}_{q}$. Observations from the spatial and temporal domains are to be processed intelligently in making the bit decisions at the desired receiver. Our goal is to minimize the total transmitted power by the system subject to the SIR constraint at each mobile that employs the linear receiver to maximize the SIR. The problem we are formulating here is the joint transmitterreceiver optimization problem over transmitter signatures $\hat{\mathbf{S}}_{q}$ and receiver filters $\tilde{\mathbf{C}}_{q}$ given by

$$
\min _{\left\{\hat{\mathbf{S}}_{q}\right\}} \sum_{q=1}^{K}\left\|\hat{\mathbf{S}}_{q}\right\|^{2}
$$

subject to

$$
\max _{\tilde{\mathbf{C}}_{q}} \frac{\left|\tilde{\mathbf{C}}_{q}^{\mathrm{H}} \mathbf{H}_{q} \hat{\mathbf{S}}_{q}\right|^{2}}{\sum_{k \neq q}^{K}\left|\tilde{\mathbf{C}}_{q}^{\mathrm{H}} \mathbf{H}_{q} \hat{\mathbf{S}}_{k}\right|^{2}+\sigma^{2}\left\|\tilde{\mathbf{C}}_{q}\right\|^{2}} \geq \gamma_{q}, \quad \forall q,
$$

where $\gamma_{q}$ is the minimum SIR requirement of user $q$.

The MMSE receiver [19] minimizes mean-squared error (MSE) and maximizes the SIR [5]. Thus, the solution to the maximization problem addressed in the constraint of (8), (9) is the MMSE receiver filter coefficients for given $\left\{\hat{\mathbf{S}}_{q}\right\}$, which is

$$
\mathbf{C}_{q}=\left[\sum_{k=1}^{K}\left(\mathbf{H}_{q} \hat{\mathbf{S}}_{k} \hat{\mathbf{S}}_{k}^{\mathrm{H}} \mathbf{H}_{q}^{\mathrm{H}}\right)+\sigma^{2} \mathbf{I}_{D}\right]^{-1} \mathbf{H}_{q} \hat{\mathbf{S}}_{q}, \quad \forall q .
$$

Assuming $\mathbf{C}_{q}$ as given in the above equation, we rewrite the optimization problem of (8), (9) as

$$
\min _{\left\{\hat{\mathbf{S}}_{q}\right\}} \sum_{q=1}^{K}\left\|\hat{\mathbf{S}}_{q}\right\|^{2}
$$


subject to

$$
\frac{\left|\mathbf{C}_{q}^{\mathrm{H}} \mathbf{H}_{q} \hat{\mathbf{S}}_{q}\right|^{2}}{\sum_{k \neq q}^{K}\left|\mathbf{C}_{q}^{\mathrm{H}} \mathbf{H}_{q} \hat{\mathbf{S}}_{k}\right|^{2}+\sigma^{2}\left\|\mathbf{C}_{q}\right\|^{2}} \geq \gamma_{q}, \quad \forall q .
$$

Now we will establish some elementary properties of the optimization problem just proposed in (11), (12). Since $\left|\mathbf{X}^{\mathrm{H}} \mathbf{Y}\right|^{2}=\left|\mathbf{Y}^{\mathrm{H}} \mathbf{X}\right|^{2}$ for any two vectors $\mathbf{X}$ and $\mathbf{Y}$ of the same size, denoting $\overline{\mathbf{C}}_{q}^{\mathrm{H}}=\mathbf{C}_{q}^{\mathrm{H}} \mathbf{H}_{q}$ and $\sigma_{q}^{2}=\sigma^{2}\left\|\mathbf{C}_{q}\right\|^{2}$, the optimization problem in (11), (12) can be formulated as

$$
\min _{\left\{\hat{\mathbf{S}}_{q}\right\}} \sum_{q=1}^{K}\left\|\hat{\mathbf{S}}_{q}\right\|^{2}
$$

subject to

$$
\gamma_{q}\left(\sum_{k \neq q}^{K}\left|\hat{\mathbf{S}}_{k}^{\mathrm{H}} \overline{\mathbf{C}}_{q}\right|^{2}+\sigma_{q}^{2}\right)-\left|\hat{\mathbf{S}}_{q}^{\mathrm{H}} \overline{\mathbf{C}}_{q}\right|^{2} \leq 0, \quad \forall q .
$$

For given $\mathbf{C}_{q}$ or $\overline{\mathbf{C}}_{q}$, we recognize that the above optimization problem is similar to the optimization problem proposed in [13] to find the optimum (may not be global optimum) transmit beamforming weights for time division multiple access (TDMA) type of systems. The optimum solution is obtained with equality in (13), (14). In [14] it is shown that if the optimization problem proposed in (13), (14) is feasible, then the algorithm in [13], applied to an appropriately scaled version of the problem, will converge to the global minimum of the original optimization problem.

The algorithm that converges to a fixed point of the desired minimization problem proposed in (8), (9) is called $o p$ timization algorithm or $O A$. We find that the convergence of the OA is a special case of the convergence of the constrained optimization algorithm, which will be proposed in the following subsection.

\subsection{Fixed transmitter temporal spreading sequences}

In the downlink of the existing IS-95, users' spreading sequences are generated by multiplying the Walsh codes with unique spreading sequences of the base station. Thus, CDMA systems, similar to IS-95, may not allow us to change the transmitter temporal sequences. For this case, we propose to maximize the system capacity by designing the optimum spatial sequences (or antenna weights) and the MMSE receiver for each user. We call the algorithm that solves this constrained optimization problem as optimization algorithm with fixed spreading sequence or OAS. The OAS is presented in Scheme 1 and its proof is given in Section 4 . Of course, due to the constraint on temporal sequences, the solution provided by the OAS will be suboptimum with respect to the solution given by the OA. Without loss of generality, we can assume that under the OAS, the temporal sequences at the transmitters are normalized to unity.

Letting $\left\{\mathbf{A}_{q}\right\}$ as an identity matrix of size $M L \times M L$ and changing $\mathbf{w}_{q}$ from $M \times 1$ to $M L \times 1$ and then denoting $\mathbf{S}_{q}=\mathbf{w}_{q}$ in Step 4 of the OAS, we will obtain the OA. In Section 4 , we
Step 1. Set $n=1$ and choose nonzero initial temporal-spatial sequences $\left\{\mathbf{S}_{q}(n)\right\}$.

Step 2. Derive MMSE receiver

$$
\mathbf{C}_{q}(n)=\left[\sum_{k=1}^{K}\left(\mathbf{H}_{q} \mathbf{S}_{k}(n) \mathbf{S}_{k}^{\mathrm{H}}(n) \mathbf{H}_{q}^{\mathrm{H}}\right)+\sigma^{2} \mathbf{I}_{D}\right]^{-1} \mathbf{H}_{q} \mathbf{S}_{q}(n), \quad \forall q .
$$

Step 3. Equate

$$
\begin{aligned}
\overline{\mathbf{C}}_{q}^{\mathrm{H}}(n) & =\mathbf{C}_{q}^{\mathrm{H}}(n) \mathbf{H}_{q}, \\
\sigma_{q}^{2}(n) & =\sigma^{2}\left\|\mathbf{C}_{q}(n)\right\|^{2}, \quad \forall q .
\end{aligned}
$$

Step 4. Set $n=n+1$ and select joint spatial sequences and powers at the transmitter

$$
\left\{\mathbf{w}_{q}(n)\right\}=\arg \min _{\left\{\hat{\mathbf{w}}_{q}\right\}} \sum_{q=1}^{K}\left\|\hat{\mathbf{w}}_{q}\right\|^{2}
$$

such that

$$
\begin{aligned}
\gamma_{q}\left(\sum_{k \neq q}^{K}\left|\hat{\mathbf{w}}_{k}^{\mathrm{H}} \mathbf{A}_{k}^{\mathrm{H}} \overline{\mathbf{C}}_{q}(n-1)\right|^{2}+\sigma_{q}^{2}(n-1)\right) \\
-\left|\hat{\mathbf{w}}_{q}^{\mathrm{H}} \mathbf{A}_{q}^{\mathrm{H}} \overline{\mathbf{C}}_{q}(n-1)\right|^{2} \leq 0, \quad \forall q .
\end{aligned}
$$

Step 5. Derive the joint temporal-spatial sequences

$$
\mathbf{S}_{q}(n)=\mathbf{A}_{q} \mathbf{w}_{q}(n), \quad \forall q
$$

and Go to Step 2.

Scheme 1: Optimization algorithm with fixed spreading sequence (OAS).

present the algorithm that solves the optimization problem of Step 4 as well as prove its convergence.

\subsection{Optimum transmit and receive beamforming}

In this paper, our assumption is that the transmitter will inform receivers of their filter coefficients. In practice, the number of filter coefficients or the length of $\mathbf{C}_{q}$ will be reasonably large and moreover, those coefficients are complex. As a result, this feedback transmission will reduce the effective transmitter-receiver throughput, which may not be desirable. In addition, complexities in constructing linear MMSE receivers at each iteration at Step 2 may not be manageable. Thus, in practice, we may come across constraints not only on the temporal sequences but also on feeding back to or constructing receiver coefficients for users. To handle this problem, we propose the following receiver structure for user $q$ :

$$
\mathbf{C}_{q}=\mathbf{H}_{q} \mathbf{A}_{q} \mathbf{Z}_{q}
$$

where the matrix $\mathbf{A}_{q}$ contains the normalized temporal sequences for user $q$ as described in (2). The term $\mathbf{Z}_{q}$, a vector of size $M \times 1$, is the receive beamforming weights (or spatial 
receiver filter) of receiver $q$ and is the solution to

$$
\mathbf{Z}_{q}=\arg \min _{\hat{\mathbf{Z}}_{q}} E\left[\left|\hat{\mathbf{Z}}_{q}^{\mathrm{H}} \mathbf{A}_{q}^{\mathrm{H}} \mathbf{H}_{q}^{\mathrm{H}} \mathbf{R}_{q}-b_{q}\right|^{2}\right] .
$$

Denoting $\mathbf{B}_{q, k}=\mathbf{A}_{q}^{\mathrm{H}} \mathbf{H}_{q}^{\mathrm{H}} \mathbf{H}_{q} \mathbf{A}_{k}$ as an $M \times M$ matrix, we can write the desired receiver structure as

$$
\begin{aligned}
\mathbf{C}_{q} & =\mathbf{H}_{q} \mathbf{A}_{q} \mathbf{Z}_{q} \\
& =\mathbf{H}_{q} \mathbf{A}_{q}\left[\sum_{k=1}^{K}\left(\mathbf{B}_{q, k} \mathbf{w}_{k} \mathbf{w}_{k}^{\mathrm{H}} \mathbf{B}_{q, k}^{\mathrm{H}}\right)+\sigma^{2} \mathbf{B}_{q, q}\right]^{-1} \mathbf{B}_{q, q} \mathbf{w}_{q} .
\end{aligned}
$$

The receiver proposed in (15) has been developed by employing optimal weights on the received fixed temporal sequences. This receiver is a constrained linear MMSE receiver. We prove in the following section that the MMSE receiver maximizes the SIR in the unconstrained as well as in the constrained filter spaces as shown in (15). This result will permit us to claim that if we develop an algorithm similar to the OAS, it will converge to a fixed point where the antenna weights and the structure of the linear receiver will be jointly optimized. Since this algorithm will optimally develop the beamforming weights at both the transmitter and receiver ends, we call this optimization algorithm the optimum beamforming algorithm (OBA). The difference between the OBA and OAS, is that the OBA uses constrained MMSE receiver in Step 2, whereas the OAS employs the unconstrained MMSE receiver.

\section{CONVERGENCE OF ALGORITHMS}

In this section, we prove the convergence of the proposed algorithms. To do so, we first prove the following lemma, which provides the property of the receiver structures in Step 2.

Lemma 1. The unconstrained MMSE receiver of (10) satisfies

$$
\begin{aligned}
\mathbf{C}_{q} & =\left[\sum_{k=1}^{K}\left(\mathbf{H}_{q} \hat{\mathbf{S}}_{k} \hat{\mathbf{S}}_{k}^{\mathrm{H}} \mathbf{H}_{q}^{\mathrm{H}}\right)+\sigma^{2} \mathbf{I}_{D}\right]^{-1} \mathbf{H}_{q} \hat{\mathbf{S}}_{q} \\
& =\alpha_{q}\left[\sum_{k \neq q}^{K}\left(\mathbf{H}_{q} \hat{\mathbf{S}}_{k} \hat{\mathbf{S}}_{k}^{\mathrm{H}} \mathbf{H}_{q}^{\mathrm{H}}\right)+\sigma^{2} \mathbf{I}_{D}\right]^{-1} \mathbf{H}_{q} \hat{\mathbf{S}}_{q}
\end{aligned}
$$

where

$$
\alpha_{q}=\frac{1}{1+\hat{\mathbf{S}}_{q}^{\mathrm{H}} \mathbf{H}_{q}^{\mathrm{H}}\left[\sum_{k \neq q}^{K}\left(\mathbf{H}_{q} \hat{\mathbf{S}}_{k} \hat{\mathbf{S}}_{k}^{\mathrm{H}} \mathbf{H}_{q}^{\mathrm{H}}\right)+\sigma^{2} \mathbf{I}_{D}\right]^{-1} \mathbf{H}_{q} \hat{\mathbf{S}}_{q}}
$$

and similarly, the constrained MMSE receiver of (17) satisfies

$$
\begin{aligned}
\mathbf{C}_{q} & =\mathbf{H}_{q} \mathbf{A}_{q}\left[\sum_{k=1}^{K}\left(\mathbf{B}_{q, k} \mathbf{w}_{k} \mathbf{w}_{k}^{\mathrm{H}} \mathbf{B}_{q, k}^{\mathrm{H}}\right)+\sigma^{2} \mathbf{B}_{q, q}\right]^{-1} \mathbf{B}_{q, q} \mathbf{w}_{q} \\
& =\beta_{q} \mathbf{H}_{q} \mathbf{A}_{q}\left[\sum_{k \neq q}^{K}\left(\mathbf{B}_{q, k} \mathbf{w}_{k} \mathbf{w}_{k}^{\mathrm{H}} \mathbf{B}_{q, k}^{\mathrm{H}}\right)+\sigma^{2} \mathbf{B}_{q, q}\right]^{-1} \mathbf{B}_{q, q} \mathbf{w}_{q},
\end{aligned}
$$

where

$$
\beta_{q}=\frac{1}{1+\mathbf{w}_{q}^{\mathrm{H}} \mathbf{B}_{q, q}^{\mathrm{H}}\left[\sum_{k \neq q}^{K}\left(\mathbf{B}_{q, k} \mathbf{w}_{k} \mathbf{w}_{k}^{\mathrm{H}} \mathbf{B}_{q, k}^{\mathrm{H}}\right)+\sigma^{2} \mathbf{B}_{q, q}\right]^{-1} \mathbf{B}_{q, q} \mathbf{w}_{q}} .
$$

In order to establish the convergence of the proposed algorithms we will need the following proposition.

Proposition 1. A linear receiver maximizes the SIR in the unconstrained or constrained filter space if and only if that receiver is the MMSE receiver or its scaled version in the corresponding filter space.

Now we concentrate on Step 4, where we are seeking optimum transmitters for users given that users' receiver structures are fixed. Recall that Step 4 of the OAS is the same as that of the OBA and is a generalization to that of the OA. Thus, we will concentrate on solving the optimization problem proposed in Step 4 of the OAS. To solve this optimization problem, we extend the algorithm of [14], which yields optimum downlink beamforming weights (i.e, spatial sequences) for SDMA systems to CDMA systems. Note that at each iteration of the OA, Step 4 will yield optimum joint temporalspatial sequences for given linear receivers derived in Step 2, whereas, the OAS and the OBA generate optimum spatial sequences for the corresponding linear receivers in the downlink.

Normalizing $\hat{\mathbf{C}}_{q}=\overline{\mathbf{C}}_{q} / \sigma_{q}$ for all $q$, we formulate the desired downlink constrained optimization problem as

$$
\min _{\left\{\mathbf{w}_{q}\right\}} \sum_{q=1}^{K}\left\|\mathbf{w}_{q}\right\|^{2}
$$

subject to

$$
\gamma_{q}\left(\sum_{k \neq q}^{K}\left|\mathbf{w}_{k}^{\mathrm{H}} \mathbf{A}_{k}^{\mathrm{H}} \hat{\mathbf{C}}_{q}\right|^{2}+1\right)-\left|\mathbf{w}_{q}^{\mathrm{H}} \mathbf{A}_{q}^{\mathrm{H}} \hat{\mathbf{C}}_{q}\right|^{2} \leq 0, \quad \forall q .
$$

In problem (22), (23), it can be shown that there exists a global minimum, if a feasible solution exists. Moreover, by contradiction we find that at the global minimum, the constraints in (22), (23) are satisfied with equality. Normalizing the downlink spatial sequences $\mathbf{w}_{q}$ such that $\mathbf{w}_{q}=\sqrt{ } \bar{P}_{q} \mathbf{W}_{q}$ where $\bar{P}_{q}$ is a nonzero scalar and $\mathbf{W}_{q}^{\mathrm{H}} \mathbf{A}_{q}^{\mathrm{H}} \hat{\mathbf{C}}_{q}=1$, we obtain the following lemma.

Lemma 2. At the global minimum of the downlink problem, the following set of equations will be satisfied for all q:

$$
\begin{gathered}
\mathbf{W}_{q}-\lambda_{q} \mathbf{A}_{q}^{\mathrm{H}} \hat{\mathbf{C}}_{q}+\sum_{k \neq q}^{K} \lambda_{k} \gamma_{k} \mathbf{A}_{q}^{\mathrm{H}} \hat{\mathbf{C}}_{k} \hat{\mathbf{C}}_{k}^{\mathrm{H}} \mathbf{A}_{q} \mathbf{W}_{q}=\mathbf{0}, \\
\mathbf{W}_{q}^{\mathrm{H}} \mathbf{A}_{q}^{\mathrm{H}} \hat{\mathbf{C}}_{q}=1 \\
\gamma_{q}\left(\sum_{k \neq q}^{K} \bar{P}_{k}\left|\mathbf{W}_{k}^{\mathrm{H}} \mathbf{A}_{k}^{\mathrm{H}} \hat{\mathbf{C}}_{q}\right|^{2}+1\right)=\bar{P}_{q}
\end{gathered}
$$

where $\left\{\lambda_{q}\right\}$ are Lagrangian coefficients. 
The proof of the above lemma is given in the appendix. Now we show that the optimal transmit spatial sequences of the downlink are a scaled version of the receive filter coefficients for the virtual uplink problem. One iteration for the downlink optimization algorithm consists of one iteration for virtual uplink optimization algorithm. In fact, the feasibility of the virtual uplink problem and that of the downlink problem are equivalent [13]. In the virtual uplink problem, the mobiles seek to minimize the total transmitter powers subject to their SIR requirements. Here, user $q$ uses $\hat{\mathbf{C}}_{q}$ as its spreading sequences and the base station employs a linear receiver, which maximizes the SIR in the constrained filter spaces. The problem, which we would like to solve now, is as follows:

$$
\min _{\left\{P_{q}\right\}} \sum_{q=1}^{K} P_{q}\left|\hat{\mathbf{C}}_{q}\right|^{2}
$$

subject to

$$
\max _{\mathbf{w}_{q}} \frac{P_{q}\left|\mathbf{w}_{q}^{\mathrm{H}} \mathbf{A}_{q}^{\mathrm{H}} \hat{\mathbf{C}}_{q}\right|^{2}}{\sum_{k \neq q}^{K} P_{k}\left|\mathbf{w}_{q}^{\mathrm{H}} \mathbf{A}_{q}^{\mathrm{H}} \hat{\mathbf{C}}_{k}\right|^{2}+\left|\mathbf{w}_{q}\right|^{2}} \geq \gamma_{q}
$$

subject to

$$
\left|\mathbf{w}_{q}^{\mathrm{H}} \mathbf{A}_{q}^{\mathrm{H}} \hat{\mathbf{C}}_{q}\right|^{2}=1, \quad \forall q .
$$

Recall that $\hat{\mathbf{C}}_{q}$ is derived from the downlink receiver of user $q$ after processing as $\mathbf{H}_{q}^{\mathrm{H}} \mathbf{C}_{q} / \sigma_{q}$. We can view $\mathbf{A}_{q} \mathbf{w}_{q}$ as a temporal-spatial filter of user $q$, where $\mathbf{A}_{q}$ is the temporal filter and $\mathbf{w}_{q}$ is the spatial filter. Also note that $\gamma_{q}$ is the same in both the uplink and the downlink problems.

We can show by contradiction that at the global minimum of the above optimization problem, the constraint will be satisfied with equality and the optimum power vector is component-wise less than or equal to any feasible power vector of the optimization problem. Thus, the power vector, which will be optimum to problem (26), (27), and (28), will also be the optimum solution if we change the cost function of the above problem as $\sum_{q=1}^{K} P_{q}$. Now we state a proposition, which yields an algorithm to solve the optimization problem of the virtual uplink with the assumption that it is feasible.

Proposition 2. The following iterative algorithm has a unique fixed point, thus, it is the global minimum of the virtual uplink problem:

$$
P_{q}(n+1)=\gamma_{q} \min _{\mathbf{w}_{q}}\left(\sum_{k \neq q}^{K} P_{k}(n)\left|\mathbf{w}_{q}^{\mathrm{H}} \mathbf{A}_{q}^{\mathrm{H}} \hat{\mathbf{C}}_{k}\right|^{2}+\left|\mathbf{w}_{q}\right|^{2}\right)
$$

subject to

$$
\left|\mathbf{w}_{q}^{\mathrm{H}} \mathbf{A}_{q}^{\mathrm{H}} \hat{\mathbf{C}}_{q}\right|^{2}=1 .
$$

The proof of Proposition 2 follows from the framework of standard interference functions developed in [20]. The convergence of the above type of algorithms has been proven using the properties of standard interference functions in
$[3,5,14]$. Now we present some properties of the transmitter powers $\left\{P_{q}\right\}$ and the spatial filter $\left\{\mathbf{w}_{q}\right\}$ of user $q$ at the global minimum of the uplink problem.

Lemma 3. The transmitter powers $\left\{P_{q}\right\}$ and the spatial filters $\left\{\mathbf{w}_{q}\right\}$ at the global minimum of the uplink problem will satisfy the following set of equations for all $q$ :

$$
\begin{gathered}
\mathbf{w}_{q}-\frac{P_{q}}{\gamma_{q}} \mathbf{A}_{q}^{\mathrm{H}} \hat{\mathbf{C}}_{q}+\sum_{k \neq q} P_{k} \mathbf{A}_{q}^{\mathrm{H}} \hat{\mathbf{C}}_{k} \hat{\mathbf{C}}_{k}^{\mathrm{H}} \mathbf{A}_{q} \mathbf{w}_{q}=0, \\
\mathbf{w}_{q}^{\mathrm{H}} \mathbf{A}_{q}^{\mathrm{H}} \hat{\mathbf{C}}_{q}=1 .
\end{gathered}
$$

The proof of the above lemma will be found in the appendix. Employing a similar technique used in [14], we obtain the following lemma.

Lemma 4. If the transmitter powers $\left\{p_{q}\right\}$ and the spatial filters $\left\{\boldsymbol{\omega}_{q}\right\}$ are the solutions to (31) and (32), then they are unique.

The above lemma implies that (31) and (32) will be satisfied only by the global minimum of the virtual uplink problem, if that is feasible. If we denote $\lambda_{q} \gamma_{q}=P_{q}$ for all $q$ in (31) and compare (31) and (32) with (24), then from Lemma 4, we get the following two lemmas.

Lemma 5. Any normalized spatial sequences that satisfy (24) will be unique.

Lemma 6. The global optimum spatial filters $\left\{\mathbf{w}_{o, q}\right\}$ of the uplink problem are the global optimum normalized spatial sequences $\left\{\mathbf{W}_{o, q}\right\}$ in the downlink problem.

Using Lemma 6, we claim that the iterative algorithm in Proposition 2 yields the global optimum normalized spatial sequences not only for the uplink problem but also for the downlink problem. In Scheme 2, we present the algorithm that solves the optimization problem in Step 4 of the OAS for all $q$. Now we conclude the convergence of the algorithm in Step 4 by the following remarks:

(1) Proposition 2 implies that Step 4d of Scheme 2 will converge to the global optimum transmitter powers and the spatial filter coefficients $\left\{\mathbf{w}_{o, q}\right\}$ of the virtual uplink;

(2) Lemma 6 states that the optimum spatial filters $\left\{\mathbf{w}_{o, q}\right\}$ of the uplink problem are the global optimum normalized spatial sequences $\left\{\mathbf{W}_{o, q}\right\}$ in the downlink problem;

(3) once Step 4d converges, the convergence of Step 4 to the global minimum will be ensured by the convergence of Step 4f, which is guaranteed by the following proposition.

Proposition 3. If the downlink problem is feasible, then

$$
\bar{P}_{q}(m+1)=\gamma_{q}\left(\sum_{k \neq q}^{K} \bar{P}_{k}(m)\left|\mathbf{W}_{o, k}^{\mathrm{H}} \mathbf{A}_{k}^{\mathrm{H}} \mathbf{c}_{q}\right|^{2}+1\right), \quad \forall q
$$

will converge to a unique fixed point. 
Step 4a. Normalize $\overline{\mathbf{C}}_{q}(n)$ by $\sigma_{q}$ as $\hat{\mathbf{C}}_{q}(n)=\overline{\mathbf{C}}_{q}(n) / \sigma_{q}$ for all $q$

Step $4 \mathrm{~b}$. Set $m=1$ and denote $\mathbf{c}_{q}=\hat{\mathbf{C}}_{q}(n)$ for all $q$

Step 4c. Initialize $P_{q}(m) \neq 0$ and $\bar{P}_{q}(m) \neq 0$ for all $q$

Step 4d. Update the spatial filters and transmitter powers of the virtual uplink problem

$$
\overline{\mathbf{w}}_{q}(m)=\arg \min _{\hat{\mathbf{w}}_{q}}\left(\sum_{k \neq q}^{K} P_{k}(m)\left|\hat{\mathbf{w}}_{q}^{\mathrm{H}} \mathbf{A}_{q}^{\mathrm{H}} \mathbf{c}_{k}\right|^{2}+\left|\hat{\mathbf{w}}_{q}\right|^{2}\right)
$$

such that

$$
\hat{\mathbf{w}}_{q}^{\mathrm{H}} \mathbf{A}_{q}^{\mathrm{H}} \mathbf{c}_{q}=1 \quad \forall q
$$

$$
\begin{aligned}
& P_{q}(m+1) \\
& \quad=\gamma_{q}\left(\sum_{k \neq q}^{K} P_{k}(m)\left|\overline{\mathbf{w}}_{q}^{\mathrm{H}}(m) \mathbf{A}_{q}^{\mathrm{H}} \mathbf{c}_{k}\right|^{2}+\left|\overline{\mathbf{w}}_{q}(m)\right|^{2}\right) \quad \forall q
\end{aligned}
$$

Step 4e. Derive spatial filters $\mathbf{W}_{q}(m)=\overline{\mathbf{w}}_{q}(m)$ for all $q$

Step 4 f. Scale the spatial filters of the downlink problem

$$
\bar{P}_{q}(m+1)=\gamma_{q}\left(\sum_{k \neq q}^{K} \bar{P}_{k}(m)\left|\mathbf{W}_{k}^{\mathrm{H}}(m) \mathbf{A}_{k}^{\mathrm{H}} \mathbf{c}_{q}\right|^{2}+1\right) \quad \forall q
$$

Step $4 \mathrm{~g}$. Set $m=m+1$ and Go to Step $4 \mathrm{~d}$.

Scheme 2: This algorithm solves the optimization problem of Step 4 of the OAS algorithm.

The proof of Proposition 3 simply follows from the framework of standard interference functions developed in [20].

Now we are about to conclude the convergence of the proposed algorithms. To do so, we will need the following lemma. The proof of this lemma straightforwardly follows from the fact that the constraint in the optimization problem of Step 4 does not alter if we multiply $\mathbf{C}_{q}$ by a complex constant $\alpha$.

Lemma 7. The solution to the optimization problem in Step 4 will be the same if the receiver $\mathbf{C}_{q}$ is scaled by a complex factor $\alpha$.

The MMSE receivers are unique and maximize the SIR of all users in Step 2. This fact with Proposition 1 and Lemma 7 help us to prove, in the appendix, the following proposition.

Proposition 4. Each of the three proposed algorithms will converge to a fixed point, which is not necessarily the global optimum of the desired minimization problem.

\section{EMPIRICAL RESULTS}

To observe the performance of the OA, OAS, and OBA, an empirical study was performed with a single circular cell DS-

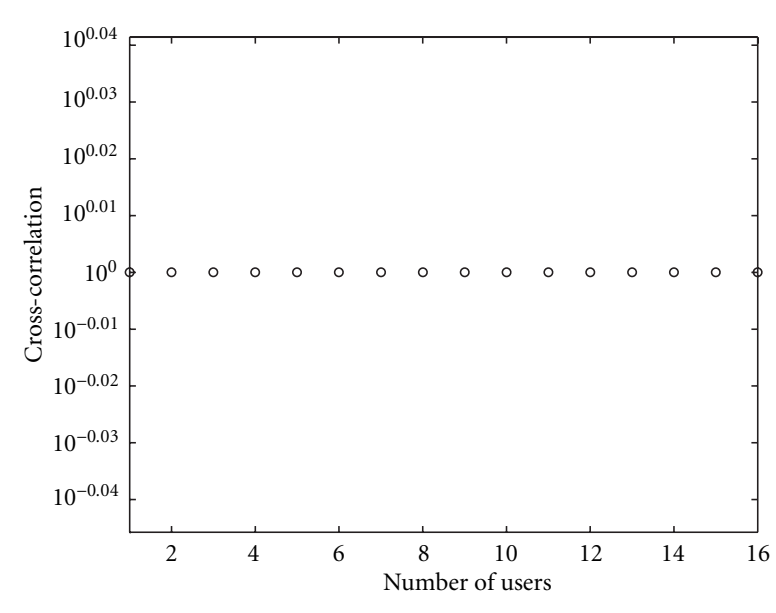

FIGURE 1: The cross-correlations between the normalized receiver filter of user 1 and its normalized received temporal-spreading sequences is shown.

CDMA system. The radius of the cell was $r_{0}=1000$ meters. Mobiles were uniformly distributed within the cell. This assumption yielded a probability density function $f(r)=2 r / r_{0}^{2}$ for the distance of a user from the base station. The path loss exponent was 4 . The height of the base station was 30 meters so that the downlink channel gain to a user from the base station was $h=1 /\left(r^{2}+30^{2}\right)^{2}$. The number of transmitting antennas at the base station was 2 . Transmitted pulses were rectangular. Transmitted signal of each antenna was received by a user over a multipath channel, where the number of paths was two and the path delays were 0 and $T_{c}$. We used $T_{c}=1$. Channel coefficients were modeled as independent identically distributed complex Gaussian random variables with mean zero and mean square value $h / 2$. The processing gain of the system was 16 . To implement the OAS and OBA, we used the same Walsh code for each user at both antennas. The spreading sequences of different users were derived by multiplying a unique spreading sequence with different orthogonal Walsh codes. We assumed that each of the three algorithms converged, when $\min _{q}\left(\left|\gamma_{q, 2}(n)-\gamma_{q}\right| / \gamma_{q}\right)<0.00001$, where $\gamma_{q, 2}(n)$ is the SIR of user $q$ at iteration $n$ immediately after Step 2. The algorithm of Step 4 was assumed to be converged when $|\mathrm{TP}(n)-\operatorname{TP}(n+1)| / \operatorname{TP}(n)<0.00001$, where $\operatorname{TP}(n)$ was the total transmitter power by the downlink system at iteration $n$. The back-ground noise variance was $\sigma^{2}=6 \times 10^{-14}$. The target SIR of all users were $10 \mathrm{~dB}$.

Figure 1 shows $\left(\mathbf{C}_{1}^{\mathrm{H}} /\left\|\mathbf{C}_{1}\right\|\right)\left(\mathbf{H}_{1} \mathbf{S}_{1} /\left\|\mathbf{H}_{1} \mathbf{S}_{1}\right\|\right)$, which is the cross-correlations between the normalized receiver filter of user 1 and its normalized received temporal-spreading sequences as a function of the number of users. We observed that under the OA, the linear MMSE receiver matches the received transmitter sequences. Recently [9] identifies that in a synchronous CDMA system, the linear MMSE receiver is the matched filter under the optimum transmitter sequences $[9,11]$ and powers. In the synchronous CDMA system, the optimum signature sequences minimize the total squared correlation (TSC); they form a set of orthogonal sequences, if the number of users is less than or equal to the processing 

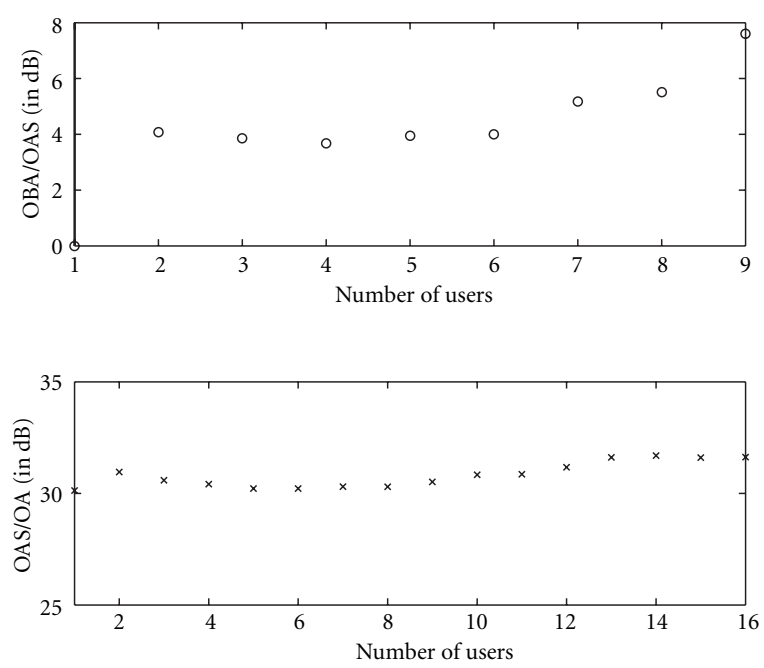

FIgURE 2: The relative performance of the algorithms is shown.

gain, and a set of WBE sequences otherwise. The results of [9] motivate [21] to design a linear receiver for multi-inputs and multi-outputs (MIMO) dispersive communication channels, whose coefficients matches the efficient transmitted transmitter sequences.

In the second experiment, we performed a simulation study to see how the OA, OAS, and OBA perform with respect to each other. Figure 2 shows the relative performance of the algorithms where the total transmitter power subject to SIR requirement by the system was taken as the performance metric. In this experiment, we observed that the OBA became infeasible when the number of users was greater than 9. Our experimental result suggested that the OA is far superior to the OAS, whereas the OAS significantly outperformed the OBA. This result is well expected and can be explained as follows. The OA takes advantage of both the temporal and spatial diversities at both the transmitter and receiver ends. However, unlike the OA, OAS is constrained by the fixed transmitter temporal sequences. Although both the OAS and OBA use the same temporal spreading sequences, the better performance of the OAS than that of the OBA is due to the fact that the OBA uses constrained MMSE receiver, whereas the OAS employs the unconstrained MMSE receiver.

\section{CONCLUSION}

Next generation wireless systems are being designed to support both voice and high capacity flexible data services through available limited bandwidths. Interference and multipath fading inherent to the wireless link make this a difficult task. However, future wireless systems must adapt to this adverse radio environment efficiently. This situation leads us to develop the joint transmitter-receiver optimization algorithms for the next generation CDMA systems under various system circumstances where we minimize the total power transmitted by the system subject to SIR requirements of users. First, we develop the optimization algorithm con- sidering no constraint on the system and referred to it as OA. Afterwards, taking practical system constraints on the transmitter-receiver structures into account, we propose two joint transmitter-receiver optimization algorithms and call them as OAS and OBA. Both the OAS and OBA are constrained by the fixed temporal spreading sequences, however, the OAS employs the unconstrained MMSE receiver, whereas the OBA employs the constrained MMSE receiver. We prove the convergence of all the proposed three algorithms. In our experiments, we observed that under the OA, the optimum receiver structure matches the received transmitter sequences. Our empirical results also indicated that the OA is far superior to the OAS in terms of performance, whereas the OAS outperformed the OBA significantly.

\section{APPENDIX}

Proof of Lemma 1. Matrix inversion lemma [5] states that an invertible matrix $\mathbf{M}$ and vectors $\mathbf{u}$ and $\mathbf{v}$ satisfy the following equality:

$$
\left(\mathbf{M}+\mathbf{u} \mathbf{v}^{\mathrm{H}}\right)^{-1}=\mathbf{M}^{-1}-\frac{\mathbf{M}^{-1} \mathbf{u} \mathbf{v}^{\mathrm{H}} \mathbf{M}^{-1}}{1+\mathbf{v}^{\mathrm{H}} \mathbf{M}^{-1} \mathbf{u}} .
$$

In (10), if we assume that

$$
\begin{aligned}
& \mathbf{M}=\sum_{k \neq q}^{K}\left(\mathbf{H}_{q} \hat{\mathbf{S}}_{k} \hat{\mathbf{S}}_{k}^{\mathrm{H}} \mathbf{H}_{q}^{\mathrm{H}}\right)+\sigma^{2} \mathbf{I}_{D}, \\
& \mathbf{u}=\mathbf{v}=\mathbf{H}_{q} \hat{\mathbf{S}}_{q},
\end{aligned}
$$

then (A.1) yields

$$
\begin{aligned}
\mathbf{C}_{q} & =\mathbf{M}^{-1} \mathbf{u}-\frac{\mathbf{M}^{-1} \mathbf{u} \mathbf{u}^{\mathrm{H}} \mathbf{M}^{-1} \mathbf{u}}{1+\mathbf{u}^{\mathrm{H}} \mathbf{M}^{-1} \mathbf{u}} \\
& =\mathbf{M}^{-1} \mathbf{u}\left(\mathbf{I}-\frac{\mathbf{u}^{\mathrm{H}} \mathbf{M}^{-1} \mathbf{u}}{1+\mathbf{u}^{\mathrm{H}} \mathbf{M}^{-1} \mathbf{u}} \mathbf{I}\right)=\frac{1}{1+\mathbf{u}^{\mathrm{H}} \mathbf{M}^{-1} \mathbf{u}} \mathbf{M}^{-1} \mathbf{u} .
\end{aligned}
$$

Using a similar method, we can show that the constrained MMSE receiver of (17) satisfies (20).

Proof of Proposition 1. In order to prove this proposition, first we show that the MMSE receiver and its scaled version maximize the SIR in the unconstrained filter spaces as well as in the constrained spatial-temporal filter spaces. The MMSE receiver [19] minimizes mean-squared error (MSE) and maximizes the SIR $[5,22]$ in the unconstrained filter spaces. Now, we prove that the MMSE receiver maximizes the SIR in the constrained spatial filter spaces as proposed in (15). For the receiver filter $\hat{\mathbf{C}}_{q}=\mathbf{H}_{q} \mathbf{A}_{q} \hat{\mathbf{Z}}_{q}$, the SIR of user $q$ is

$$
\gamma_{q}\left(\hat{\mathbf{Z}}_{q}\right)=\frac{\left|\hat{\mathbf{Z}}_{q}^{\mathrm{H}} \mathbf{B}_{q, q} \mathbf{w}_{q}\right|^{2}}{\sum_{k \neq q}^{K}\left|\hat{\mathbf{Z}}_{q}^{\mathrm{H}} \mathbf{B}_{q, k} \mathbf{w}_{k}\right|^{2}+\sigma^{2} \hat{\mathbf{Z}}_{q}^{\mathrm{H}} \mathbf{B}_{q, q} \hat{\mathbf{Z}}_{q}} .
$$

Our goal is to find $\mathbf{Z}_{q}=\hat{\mathbf{Z}}_{q}$ that maximizes $\gamma_{q}\left(\hat{\mathbf{Z}}_{q}\right)$, that 
is,

$$
\mathbf{Z}_{q}=\arg \max _{\hat{\mathbf{Z}}_{q}}\left(\gamma_{q}\left(\hat{\mathbf{Z}}_{q}\right)\right) .
$$

However, the solution to the above optimization problem is not unique. To see that, simply observe that $\mathbf{Z}_{q}$ and $\alpha \mathbf{Z}_{q}$ will produce the same SIR, where $\alpha$ is a scalar. Thus if a linear receiver maximizes the SIR, then its scaled versions will also maximize the SIR in the constrained as well as unconstrained filter spaces.

An optimum solution to (A.5) will be obtained if we solve the optimization problem with the constraint $\hat{\mathbf{Z}}_{q}^{\mathrm{H}} \mathbf{B}_{q, q} \mathbf{w}_{q}=$ $g$, where $g$ is an arbitrary complex-valued gain. Under the constraint $\hat{\mathbf{Z}}_{q}^{\mathrm{H}} \mathbf{B}_{q, q} \mathbf{w}_{q}=g$, the maximization problem in (A.5) will be equivalent to the following minimization problem:

$$
\mathbf{Z}_{q}=\arg \min _{\hat{\mathbf{Z}}_{q}}\left(\sum_{k \neq q}^{K}\left|\hat{\mathbf{Z}}_{q} \mathbf{B}_{q, k} \mathbf{w}_{k}\right|^{2}+\sigma^{2} \hat{\mathbf{Z}}_{q}^{\mathrm{H}} \mathbf{B}_{q, q} \hat{\mathbf{Z}}_{q}\right)
$$

subject to

$$
\hat{\mathbf{Z}}_{q}^{\mathrm{H}} \mathbf{B}_{q, q} \mathbf{w}_{q}=g .
$$

We solve the above optimization problem explicitly as

$$
\mathbf{Z}_{q}=\frac{g^{*}}{\mathbf{w}_{q}^{\mathrm{H}} \mathbf{B}_{q, q}^{\mathrm{H}} \mathbf{V}_{q}} \mathbf{V}_{q},
$$

where $g^{*}$ is the conjugate of $g$ and

$$
\mathbf{V}_{q}=\left[\sum_{k \neq q}^{K}\left(\mathbf{B}_{q, k} \mathbf{w}_{k} \mathbf{w}_{k}^{\mathrm{H}} \mathbf{B}_{q, k}^{\mathrm{H}}\right)+\sigma^{2} \mathbf{B}_{q, q}\right]^{-1} \mathbf{B}_{q, q} \mathbf{w}_{q} .
$$

Letting

$$
g=\frac{\mathbf{w}_{q}^{\mathrm{H}} \mathbf{B}_{q, q}^{\mathrm{H}} \mathbf{V}_{q}}{1+\mathbf{w}_{q}^{\mathrm{H}} \mathbf{B}_{q, q}^{\mathrm{H}}\left[\sum_{k \neq q}^{K}\left(\mathbf{B}_{q, k} \mathbf{w}_{k} \mathbf{w}_{k}^{\mathrm{H}} \mathbf{B}_{q, k}^{\mathrm{H}}\right)+\sigma^{2} \mathbf{B}_{q, q}\right]^{-1} \mathbf{B}_{q, q} \mathbf{w}_{q}},
$$

we find that the MMSE receiver in (20) maximizes the SIR in the constrained filter space.

Now, we prove that any linear receiver that maximizes the SIR is either the MMSE receiver or a scaled version of the MMSE receiver. From (A.8) we see that a linear receiver, which maximizes the SIR of user $q$ and satisfies the constraint $\mathbf{C}_{q}^{\mathrm{H}} \mathbf{H}_{q} \mathbf{S}_{q}=g$, can be explicitly obtained by solving the following minimization problem:

$$
\mathbf{C}_{q}=\arg \min _{\hat{\mathbf{C}}_{q}}\left(\sum_{k \neq q}^{K}\left|\hat{\mathbf{C}}_{q}^{\mathrm{H}} \mathbf{H}_{q} \mathbf{S}_{k}\right|^{2}+\sigma^{2}\left\|\hat{\mathbf{C}}_{q}\right\|^{2}\right)
$$

subject to

$$
\hat{\mathbf{C}}_{q}^{\mathrm{H}} \mathbf{H}_{q} \mathbf{S}_{q}=g .
$$

Since the above cost function is convex, the solution to the above minimization problem is unique. We have already proved that $\mathbf{C}_{q}$ will be either the MMSE receiver or its scaled version depending on the value of $g$. Note that when $\hat{\mathbf{C}}_{q}$ is constrained to be in the form of $\mathbf{H}_{q} \mathbf{A}_{q} \hat{\mathbf{Z}}_{q}$, Problem (A.11), (A.12) is equivalent to (A.6), (A.7).

Let $\overline{\mathbf{C}}_{q}$ be a linear receiver, which maximizes user $q$ 's SIR. Without loss of generality, we can assume the projection of this receiver on the received transmitter sequences as $\overline{\mathbf{C}}_{q}^{\mathrm{H}} \mathbf{H}_{q} \mathbf{S}_{q}=g$. Note that $\overline{\mathbf{C}}_{q}$ is a feasible solution to the above optimization problem and provides the same SIR as the optimum receiver $\mathbf{C}_{q}$. Thus, $\overline{\mathbf{C}}_{q}$ is also an optimum solution. Since the optimum solution to the above minimization problem is unique, we get $\overline{\mathbf{C}}_{q}=\mathbf{C}_{q}$.

Proof of Lemma 2. The Lagrangian for the optimization problem of (22), (23) is given by

$$
\begin{aligned}
& L(\mathbf{W}, \lambda) \\
& =\sum_{q=1}^{K}\left\|\hat{\mathbf{w}}_{q}\right\|^{2} \\
& \quad+\sum_{q=1}^{K} \lambda_{q}\left(\gamma_{q}\left(\sum_{k \neq q}^{K}\left|\hat{\mathbf{w}}_{k}^{\mathrm{H}} \mathbf{A}_{k}^{\mathrm{H}} \hat{\mathbf{C}}_{q}\right|^{2}+1\right)-\left|\hat{\mathbf{w}}_{q}^{\mathrm{H}} \mathbf{A}_{q}^{\mathrm{H}} \hat{\mathbf{C}}_{q}\right|^{2}\right) .
\end{aligned}
$$

Differentiating the above equation with respect to $\mathbf{w}_{q}$, the following $K$ equations are obtained as the necessary conditions for optimality:

$$
\mathbf{w}_{q}-\lambda_{q} \mathbf{A}_{q}^{\mathrm{H}} \hat{\mathbf{C}}_{q} \hat{\mathbf{C}}_{q}^{\mathrm{H}} \mathbf{A}_{q} \mathbf{w}_{q}+\sum_{k \neq q}^{K} \lambda_{k} \gamma_{k} \mathbf{A}_{q}^{\mathrm{H}} \hat{\mathbf{C}}_{k} \hat{\mathbf{C}}_{k}^{\mathrm{H}} \mathbf{A}_{q} \mathbf{w}_{q}=\mathbf{0}, \quad \forall q .
$$

In addition, the optimal solution must satisfy the following $K$ constraints, which simply follows from the optimization problem of (22), (23):

$$
\gamma_{q}\left(\sum_{k \neq q}^{K}\left|\mathbf{w}_{k}^{\mathrm{H}} \mathbf{A}_{k}^{\mathrm{H}} \hat{\mathbf{C}}_{q}\right|^{2}+1\right)=\left|\mathbf{w}_{q}^{\mathrm{H}} \mathbf{A}_{q}^{\mathrm{H}} \hat{\mathbf{C}}_{q}\right|^{2}, \quad \forall q .
$$

Using $\mathbf{w}_{q}=\sqrt{ } \bar{P}_{q} \mathbf{W}_{q}$ where $\mathbf{W}_{q}^{\mathrm{H}} \mathbf{A}_{q}^{\mathrm{H}} \hat{\mathbf{C}}_{q}=1$ with (A.14) and (A.15), we prove the lemma.

Proof of Lemma 3. Using Lagrangian with the following optimization problem:

$$
\min _{\mathbf{w}_{q}}\left(\sum_{k \neq q}^{K} P_{k}\left|\mathbf{w}_{q}^{\mathrm{H}} \mathbf{A}_{q}^{\mathrm{H}} \hat{\mathbf{C}}_{k}\right|^{2}+\left|\mathbf{w}_{q}\right|^{2}\right)
$$

subject to

$$
\left|\mathbf{w}_{q}^{\mathrm{H}} \mathbf{A}_{q}^{\mathrm{H}} \hat{\mathbf{C}}_{q}\right|^{2}=1
$$

and then differentiating, we get the necessary condition for optimality of the optimization problem (26), (27), and (28) 
as

$$
\begin{gathered}
\mathbf{w}_{q}-\lambda_{q} \mathbf{A}_{q}^{\mathrm{H}} \hat{\mathbf{C}}_{q} \hat{\mathbf{C}}_{q}^{\mathrm{H}} \mathbf{A}_{q} \mathbf{w}_{q}+\sum_{k \neq q}^{K} P_{k} \mathbf{A}_{q}^{\mathrm{H}} \hat{\mathbf{C}}_{k} \hat{\mathbf{C}}_{k}^{\mathrm{H}} \mathbf{A}_{q} \mathbf{w}_{q}=\mathbf{0}, \\
\mathbf{w}_{q}^{\mathrm{H}} \mathbf{A}_{q}^{\mathrm{H}} \hat{\mathbf{C}}_{q}=1 .
\end{gathered}
$$

Multiplying both sides of (A.18) by $\mathbf{w}_{q}^{\mathrm{H}}$ and using (A.19), we get

$$
\lambda_{q}=\sum_{k \neq q}^{K} P_{k}\left|\mathbf{w}_{q}^{\mathrm{H}} \mathbf{A}_{q}^{\mathrm{H}} \hat{\mathbf{C}}_{k}\right|^{2}+\left|\mathbf{w}_{q}\right|^{2} .
$$

Now we use the fact that at the global minimum of the virtual uplink optimization problem, the constraint of the optimization problem (26), (27), and (28) will be satisfied with equality. Coupling this fact with (A.20), we get at the global minimum, $\lambda_{q}=P_{q} / \gamma_{q}$. Substituting $\lambda_{q}$ back to (A.18), we prove the lemma.

Proof of Proposition 4. To prove this proposition, we will start from Step 2. Let $\gamma_{q, 2}(n)$ denote the SIR of user $q$ at the $n$th iteration immediately after Step 2 , where $n>1$. Proposition 1 implies that $\mathbf{C}_{q}(n)$ will maximize the SIR of user $q$ for given transmitter sequences of all users.

Recall that Steps 4 and 5 yield the optimum transmitter sequences $\left\{\mathbf{S}_{q}(n)\right\}$ for given $\left\{\mathbf{C}_{q}(n-1)\right\}$, which are the MMSE receivers of users for the transmitter sequences $\left\{\mathbf{S}_{q}(n-1)\right\}$. The global optimum solution of the minimization problem in Step 4 satisfies the constraint with equality, and thus, $\left\{\mathbf{S}_{q}(n)\right\}$ and $\left\{\mathbf{C}_{q}(n-1)\right\}$ will provide all users SIRs $\left\{\gamma_{q}\right\}$. Since the MMSE receiver maximizes the SIR, after executing Step 2, we will get

$$
\gamma_{q, 2}(n) \geq \gamma_{q}, \quad \forall q
$$

If $\gamma_{q, 2}(n)>\gamma_{q}$ for user $q$, then it is easy to show that a feasible solution can be obtained for the minimization problem of Step 4 by reducing the power of that user $q$. Thus when $\gamma_{q, 2}(n)>\gamma_{q}$, the cost function of Step 4 will strictly decrease at its next execution. When $\gamma_{q, 2}(n)=\gamma_{q}$ for all $q$, Proposition 1 yields that $\left\{\mathbf{C}_{q}(n-1)\right\}$ are either MMSE receivers or their scaled versions for transmitter sequences $\left\{\mathbf{S}_{q}(n)\right\}$. Coupling this fact with Lemma 7 , we get

$$
\mathbf{S}_{q}(n)=\mathbf{S}_{q}(n-1), \quad \forall q
$$

Since the MMSE receivers are unique for given $\left\{\mathbf{S}_{q}(n)\right\}$, we obtain

$$
\mathbf{C}_{q}(n)=\mathbf{C}_{q}(n-1), \quad \forall q
$$

Therefore, $\gamma_{q, 2}(n)=\gamma_{q}$ for all $q$ implies that the proposed algorithms have converged to a fixed point, which is not necessarily the global optimum.

\section{ACKNOWLEDGMENT}

This work has been presented in part in the Conference on Information Science and Systems, Johns Hopkins University, March 21-23, 2001.

\section{REFERENCES}

[1] P. S. Kumar and J. Holtzman, "Power control for a spread spectrum system with multiuser receivers," in Proc. IEEE International Symposium on Personal, Indoor, and Mobile Radio Communications, vol. 3, pp. 955-959, Toronto, Canada, September 1995.

[2] M. Saquib, R. Yates, and A. Ganti, "Power control for an asynchronous multi-rate decorrelator," IEEE Trans. Communications, vol. 48, no. 5, pp. 804-812, 2000.

[3] S. Ulukus and R. Yates, "Adaptive Power Control with MMSE Multiuser Detectors," in Proc. IEEE International Conference on Communications, Montreal, Quebec, Canada, June 1997.

[4] M. Varanasi, "Power control for multiuser detection," in Proc. 30th Annual Conference on Information Sciences and Systems, pp. 866-873, Princeton University, Princeton, NJ, USA, March 1996.

[5] A. Yener, R. Yates, and S. Ulukus, "Interference management for CDMA systems through power control, multiuser detection, and beamforming," IEEE Trans. Communications, vol. 49, no. 7, pp. 1227-1239, 2001.

[6] W. M. Jang, B. R. Vojcic, and R. L. Picholtz, "Joint transmitterreceiver optimization in synchronous multiuser communications over multipath channels," IEEE Trans. Communications, vol. 46, no. 2, pp. 269-278, 1998.

[7] S. Ulukus and R. Yates, "Iterative construction of optimum signature sequence sets in synchronous CDMA systems," IEEE Transactions on Information Theory, vol. 47, no. 5, pp. 19891998, 2001.

[8] S. Ulukus and R. Yates, "User capacity of asynchronous CDMA systems with optimum signature sequences," submitted to IEEE Transactions on Information Theory, April 2001.

[9] P. Viswanath, V. Anantharam, and D. N. C. Tse, "Optimal sequences, power control, and user capacity of synchronous CDMA systems with linear MMSE multiuser receivers," IEEE Transactions on Information Theory, vol. 45, no. 6, pp. 19681983, 1999.

[10] T. F. Wong and T. M. Lok, "Transmitter adaptation in multicode DS-CDMA systems," IEEE Journal on Selected Areas in Communications, vol. 19, no. 1, pp. 69-82, 2001.

[11] M. Rupf and J. L. Massey, "Optimum sequences multisets for synchronous code-division multiple-access channels," IEEE Transactions on Information Theory, vol. 40, no. 4, pp. 12611266, 1994.

[12] J. I. Concha and S. Ulukus, "Optimization of CDMA signature sequences in multipath channels," in Proc. IEEE Vehicular Technology Conference, Rhodes, Greece, May 2001.

[13] F. Rashid-Farrokhi, R. K. J. Liu, and L. Tassiulas, "Transmit beamforming and power control for cellular wireless systems," IEEE Journal on Selected Areas in Communications, vol. 16, no. 8, pp. 1437-1450, 1998.

[14] E. Visotsky and U. Madhow, "Optimal multiuser space-time transmit filtering," Coordinated Science Laboratory, University of Illinois, Urbana, Ill, USA, 1999.

[15] Y. Liang, F. P. S. Chin, and K. J. R. Liu, "Downlink beamforming for DS-CDMA mobile radio with multimedia services," IEEE Trans. Communications, vol. 49, no. 7, pp. 1288-1298, 2001. 
[16] F. Adachi, M. Sawahashi, and H. Suda, "Wideband DS-CDMA for next-generation mobile communication systems," IEEE Communication Magazine, vol. 36, no. 9, pp. 56-69, 1998.

[17] R. H. Frenkiel and T. Imielinski, "Infostations: the joy of 'many-time, many-where' communications," WINLAB Technical Report (WINLAB-TR-119), Rutgers University, April 1996.

[18] M. Saquib, Md. H. Islam, and S. Kumar, "Power control and transmit diversity in multipath CDMA systems," in Proc. IEEE Wireless Communications and Networking Conference, Chicago, Ill, USA, September 2000.

[19] Z. Xie, R. T. Short, and C. K. Rushforth, "A family of suboptimum detectors for coherent multiuser communications," IEEE Journal on Selected Areas in Communications, vol. 8, no. 4, pp. 683-690, 1990.

[20] R. D. Yates, "A framework for uplink power control in cellular radio systems," IEEE Journal on Selected Areas in Communications, vol. 13, no. 7, pp. 1341-1347, 1995.

[21] P. Dimitrie and C. Rose, "New approach to multiple antenna systems," in Proc. Conference on Information Science and Systems, Baltimore, Md, USA, March 2001.

[22] U. Madhow and M. L. Honig, "MMSE interference suppression for direct-sequence spread-spectrum CDMA," IEEE Trans. Communications, vol. 42, no. 12, pp. 3178-3188, 1994.

Mohammad Saquib received his B.S. degree (1991) in electrical and electronics engineering from Bangladesh University of Engineering \& Technology, Bangladesh (BUET). After his baccalaureate, he worked as a System Analyst (1991-92) at the Energy Research Corporation, Danbury, CT. He received the M.S. (1995) and the Ph.D. (1998) degrees in electrical engineering from Rutgers University, New Brunswick, NJ, where

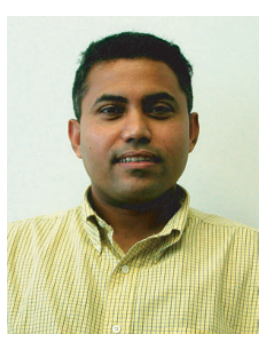
he was a Graduate Research Assistant in the Wireless Information Networks Laboratory (WINLAB). From 1998 to 1999, he was with the MIT Lincoln Laboratory, Lexington, Mass, as a member of the Technical Staff. In January 1999, he joined the Electrical and Computer Engineering Department at Louisiana State University (LSU), where he was the Donald Ceil \& Elaine T. Delaune Endowed Assistant Professor. Since July 2000, he has been with the Electrical Engineering Department at the University of Texas at Dallas (UTDallas) as Assistant Professor. His research interests include power control, interference suppression, and media access protocols for wireless communications systems.

Md Habibul Islam received his M.S. degree (1995) in electrical engineering and Masters of Business Administration (1999) from Tajik Technical University, USSR and the Institute of Business Administration, The University of Dhaka, Bangladesh, respectively. Currently, he is pursuing the Ph.D. degree in electrical engineering at The University of Texas at Dallas, Richardson, Texas. He is a Graduate Research Assistant in the

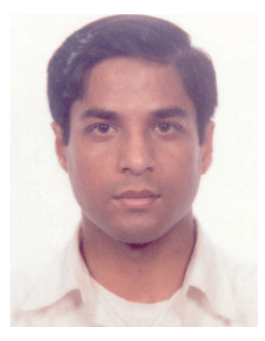
Wireless Communications Research Laboratory (WiCoRe). His research interests include power control, multiuser detection, interference management, and adaptive modulation for wireless communications systems. 